\title{
TỐI UUU CẤU HÌNH LƯớI ĐIÊN PHÂN PHỐI GIẢM TỔN THẤT CÔNG SUẤT SỬ DỤNG THUẠTT TOÁN CUCKOO SEARCH CẢI TIẾN
}

\author{
NGUYỄN THANH THUẬN, DƯƠNG THANH LONG
}

\author{
Khoa Công Nghệ Điện, Trương Đại học Công nghiệp TP. HCM; \\ nguyenthanhthuan@iuh.edu.vn; duongthanhlong@iuh.edu.vn
}

\begin{abstract}
Bài báo này trình bày phương pháp tái cấu hình lưới điện phân phối sử dụng thuật toán cuckoo search (CS). Trong đó, để nâng cao hiệu quả của thuật toán CS, cơ chế tìm kiếm cục bộ được bổ sung để khai thác vùng không gian tồn tại xung quanh cấu hình tốt nhất của quần thể trong quá trình tìm kiếm. Hàm mục tiêu của bài toán là giảm tổn thất công suất trên lưới điện phân phối. Kết quả đánh giá trên lưới điện phân phối 33 nút cho thấy phương pháp đề xuất (enhanced cuckoo search - ECS) có ưu điểm vượt trội so với phương pháp tái cấu hình dựa trên thuật toán CS thông thường về chất lượng giải pháp thu được, số lượng vòng lặp hội tụ.
\end{abstract}

Keywords. Thuật toán cuckoo search, tái cấu hình, lưới điện phân phối.

\section{OPTIMIZATION OF ELECTRIC DISTRIBUTION NETWORK CONFIGURATION FOR POWER LOSS REDUCTION USING ENHANCED CUCKOO SEARCH}

\begin{abstract}
This paper presents the method of reconfiguring the distribution network using cuckoo search algorithm (CS). In which, to improve the efficiency of CS algorithms, the local search technique is proposed to exploit the search space around the best configuration of the population during the search process. The objective function of the problem is reduction of power loss of the distribution system. The evaluated results on the distribution network with 33 nodes has showed that the enhanced cuckoo search (ECS) method outperforms compared to the reconfiguration method based on conventional CS algorithms on the solution quality obtained, the number of convergence iteration.
\end{abstract}

Keywords. cuckoo search, reconfiguration, distribution network.

\section{GIỚI THIÊU}

Lưới điện phân phối (LĐPP) thường được vận hành hở hay còn gọi là vận hành hình tia. Việc vận hành hở LĐPP có nhiều ưu điểm so với vận hành kín như là dễ dàng bảo vệ lưới, dòng sự cố nhỏ, dễ dàng điều chỉnh điện áp và phân bố công suất. Tuy nhiên, do vận hành ở mức điện áp thấp và dòng điện lớn, LĐPP thường có tổn thất công suất và độ sụt áp lớn [1]. Mặc dù được vận hành hở nhưng để nâng cao độ tin cậy của hệ thống phân phối, trên các mạch hình tia thường có các khóa điện thường mở có khả năng kết nối đến các mạch khác. Ngoài ra, trên các mạch hình tia cũng có các khóa điện thường đóng. Các khóa điện này có thể thay đổi trạng thái khi có yêu cầu. Vì vậy, trên LĐPP xuất hiện bài toán tái cấu hình LĐPP. Tái cấu hình LĐPP là quá trình thay đổi cấu trúc hình học của LĐPP bằng việc thay đổi trạng thái của các khóa điện thường đóng và thường mở trong khi vẫn đảm bảo thỏa mãn các ràng buộc tùy theo mục đích của nhà vận hành.

Bài toán tái cấu hình LĐPP được đề xuất lần đầu vào năm 1975 bởi Merlin và Back [2]. Trong nghiên cứu này, ban đầu tất cả các khóa điện được đóng lại để tạo thành lưới điện kín, sau đó các khóa điện lần lượt được mở để khôi phục lại cấu hình hình tia. Civanlar và cộng sự [3] sử dụng phương pháp trao đổi nhánh để giảm tổn thất công suất dựa trên việc chọn lựa các cạ̣p khóa điện. Ý tưởng của phương pháp là trong một vòng kín, một khóa điện đang mở sẽ được thay thế bằng một khóa đóng để giảm tổn thất công suất. Khóa được chọn là khóa có mức giảm tổn thất công suất lớn nhất. Sau gần bốn thập niên thu hút sự quan tâm của các nhà nghiên cứu, cho đến nay nhiều phương pháp tối ưu tổng quát đã được áp dụng để giải bài toán tái cấu hình LĐPP và đã đạt được nhiều kết quả. Trong [4]-[6], giải thuật di truyền (genetic algorithm - GA) đã được áp dụng giải bài toán tái cấu hình LĐPP để giảm tổn thất công suất. 
Trong [7]-[9], thuật toán tối ưu bầy đàn (Particle Swarm Optimization - PSO) được áp dụng vào bài toán tái cấu hình LĐPP để giảm tổn thất công suất. Trong [10], thuật toán bước nhảy ếch thích nghi (Adaptive Shuffled Frogs Leaping Algorithm-ASFLA) được sử dụng để giải bài toán tái cấu hình kết hợp với lắp đặt máy phát phân tán trên LĐPP. Trong [11], thuật toán tối ưu bầy đàn nhị phân chọn lọc (Selective Binary Particle Swarm Optimization (IS-BPSO) được sử dụng để giải bài toán tái cấu hình giảm tổn thất công suất. Trong [12], thuật toán tìm kiếm hài hòa (harmony search algorithm-HSA) được sử dụng để giải bài toán tái cầu hình trên LĐPP cân bằng và không cân bằng.

Có thể thấy rằng để giải bài toán tái cấu hình có 2 nhóm phương pháp chính bao gồm phương pháp heuristic và phương pháp tối ưu tổng quát: Phương pháp heuristic dựa trên các tiêu chí kỹ thuật để tìm một giải pháp tốt. Nhóm thứ hai là sử dụng các phương pháp heuristic tổng quát. Cho đến nay, đây là nhóm phương pháp hiệu quả và phổ biến nhất trong việc giải bài toán tái cấu hình. Các phương pháp này không có yêu cầu đặc biệt nào về sự khác nhau, tính liên tục, lồi lõm của hàm mục tiêu và hiệu quả trong việc xử lý các ràng buộc, tối ưu rời rạc và nhiều hướng. Tuy nhiên, những hạn chế của các phương pháp này là có thể hội tụ vào cực trị địa phương thay vì cực trị toàn cục. Do đó, mối quan tâm chính của các nhà nghiên cứu trong nhóm phương pháp này là khắc phục vấn đề hội tụ sớm của các phương pháp heuristic tổng quát.

Thuật toán CS được phát triển bởi Yang and Deb [13], ý tưởng của thuật toán dựa trên tập tính ký sinh nuôi dưỡng của một số loài chim tu hú. Đây là loài chim không có khả năng nuôi con mà chúng duy trì nòi giống bằng cách đẻ trứng nhờ trong tổ của các loài chim khác có trứng tương tự về màu sắc và kích thước. Sau khi được ấp, chim non tú hú nhanh chóng loại bỏ chim non hoặc trứng của chim khác để độc chiếm nguồn thức ăn từ chim bố mẹ nuôi. Tuy nhiên, khi đẻ nhờ trứng vào tổ của các loài chim khác, chim tu hú cũng có nguy cơ bị chim khác phát hiện ra trứng lạ và loại bỏ. Kể từ khi được đề xuất bởi Yang and Deb, CS đã được áp dụng thành công trong các lĩnh vực của hệ thống điện như bài toán điều độ thủy nhiệt điện [14], [15], [16]; bài toán tái cấu hình [17]; bài toán đặt vị trí máy phát phân tán [18], [19].

Trong bài báo này, thuật toán CS được áp dụng để giải bài toán tái cấu hình LĐPP giảm tổn thất công suất. Trong đó, cơ chế tìm kiếm cục bộ của thuật toán CS được cải tiến để khai thác được vùng không gian tổn tại xung quanh cấu hình tốt nhất hiện hữu của của quần thể. Điều này giúp cho thuật toán CS cải tiến $(\mathrm{ECS})$ tránh rơi vào cực trị địa phương và tìm được cấu trúc vận hành tối ưu với số vòng lặp nhỏ hơn so với thuật toán CS.

\section{MÔ TẢ BÀI TOÁN}

Giảm tổn thất công suất là một trong những mục tiêu quan trọng nhất của công tác vận hành LĐPP. Một trong những ưu điểm lớn nhất của biện pháp tái cấu hình LĐPP là giảm tổn thất công suất. Tổn thất công suất $(\Delta P)$ của cấu hình lưới $X^{\text {th }}$ được xác định bằng tổng tổn thất công suất trên các nhánh:

$$
\Delta P=\sum_{i=1}^{N b r} k_{i} \times R_{i} \times\left(\frac{P_{i}^{2}+Q_{i}^{2}}{V_{i}^{2}}\right)
$$

Trong đó, $N b r$ là tổng số nhánh trên lưới phân phối. $k_{i}$ bằng 0 nếu khóa điện trên nhánh $i$ mở và ngược lại bằng 1 nếu khóa điện trên nhánh $i$ đóng. $R_{i}$ là tổng trở của nhánh $i . P_{i}$ và $Q_{i}$ lần lượt là công suất tác dụng và phản kháng trên nhánh $i$. $V_{i}$ là điện áp cuối nhánh $i$.

Ngoài ra, trong quá trình tái cấu hình cần phải thõa mãn các điều kiện ràng buộc cân bằng công suất, điện áp và dòng điện nằm trong giới hạn cho phép. Do đó, hàm thích nghi của bài toán tái cấu hình được mô tả như sau:

$$
\begin{aligned}
& F i t=\sum_{i=1}^{N b r} R_{i} \times\left(\frac{P_{i}^{2}+Q_{i}^{2}}{V_{i}^{2}}\right)+K_{1} \times \max \left(V_{\text {min }, c p}-V_{\text {min }}, 0\right)+K_{2} \times \max \left(V_{\max }-V_{\text {max }, c p}, 0\right)+K_{3} \times \\
& \max \left(k_{I, \max }-k_{I, c p}, 0\right)
\end{aligned}
$$

Trong đó, $K_{l}, K_{2}$ và $K_{3}$ lần lượt là các hệ số phạt khi cấu hình lưới vi phạm các ràng buộc về thấp áp, quá áp và quá tải. max là hàm trả về giá trị lớn nhất trong tập đối số. $V_{\min , c p}$ và $V_{\max , c p}$ lần lượt là biên độ điện áp nhỏ nhất và lớn nhất cho phép được chọn bằng 0.95 p.u. và 1.05 p.u.. $V_{\min }$ và $V_{\max }$ lần lượt là biên độ điện áp nhỏ nhất và lớn nhất trong cấu hình lưới đang xét. $k_{I, \max }$ là hệ số quá tải lớn nhất trong hệ thống, $k_{I, c p}$ là hệ số quá tải cho phép trên đường dây được chọn bằng 1 . 


\section{PHƯƠNG PHÁP ĐỀ XUẤT}

Không giống như các thuật toán metaheuristic khác, thuật toán CS tạo ra hai quần thể giải pháp xem xét cho bài toán thông qua phép Lévy và phép phép phát hiện trứng lạ random walk. Trong đó, quần thể được tạo ra từ phép Lévy có tính chất khai phá không gian tìm kiếm. Trong quần thể này mỗi cá thể được tạo ra thường rất khác biệt so với cá thể tương ứng trước đó do sự phân phối ngẫu nhiên của hàm Lévy. Trong khi đó, quần thể được tạo ra từ phép phát hiện trứng lạ có tính chất khai thác không gian tìm kiếm. Đặc điểm của quần thể này, là một số cá thể được cập nhật sang vị trí mới. Tuy nhiên, việc lựa chọn các cá thể để cập nhật cũng như số biến trong một cá thể được lựa chọn để cập nhật cũng mang tính ngẫu nhiên.

Mặc dù hai cơ chế khai phá và khai thác của thuật toán CS rất hiệu quả để tìm giải pháp tối ưu. Điều này đã được chứng minh thông qua rất nhiều bài toán đã được được áp dụng thành công. Tuy nhiên, khi áp dụng giải bài toán tái cấu hình LĐPP, thuật toán CS vẫn có những hạn chế khi chưa khai thác được vùng không gian lân cận giải pháp tối ưu hiện hữu. Đây là những cấu hình LĐPP khả thi tồn tại xung quan cấu hình tốt nhất hiện hữu, chúng được tạo ra bằng việc thay đổi lần lượt từng biến điều khiển của cấu hình tốt nhất hiện hữu. Vì vậy, một cơ chế tìm kiếm cục bộ được đề xuất bổ sung cho ECS để khắc phục nhược điểm trền của CS. Chi tiết các bước thực hiện như sau:

Bước 1: Khởi tạo

Để giải bài toán tái cấu hình sử dụng thuật toán ECS, ban đầu một quần thể các cấu hình LĐPP được khởi tạo ngẫu nhiên và chúng được đánh giá hàm thích nghi bởi biểu thức (2). Quá trình mã hóa cấu trúc LĐPP và khởi tạo quần thể ban đầu được thực hiện như sau:

$$
X_{i}=\operatorname{round}\left[X_{\text {low }, j}+\text { rand } \times\left(X_{\text {high }, j}-X_{\text {low }, j}\right)\right]
$$

Trong đó, $X_{i}$ là cấu hình LĐPP thứ $i$,ới $i=1, \ldots, N$. $X_{l o w, j}=1$. $X_{h i g h, j}$ với $j=1, \ldots, D$ là vị trí lớn nhất trong vòng kín thứ $j$. Lưu ý, Vòng kín là tập hợp các khóa điện tạo thành vòng kín trên LĐPP và vòng kín thứ $j$ được tạo thành thông qua việc đóng khóa điện mở ban đầu thứ $j . N$ và $D$ lần lượt là kích thước của quần thể và số lượng khóa mở trên LĐPP.

Buớc 2: Tạo ra giải pháp mói thông qua phép di chuyển Lévy

Từ quần thể giải pháp hiện tại, một quần thể mới được tạo ra dựa trên phép Lévy. Nếu từng cá thể trong quần thể mới có sự cải thiện trong hàm thích nghi so với cá thể tương ứng trong quần thể hiện tại, thì các cá thể này được cập nhật vào quần thể hiện tại. Quá trình tạo ra quần thể mới sử dụng phép Lévy được thực hiện như sau:

$$
X_{i}^{\text {new }}=\operatorname{round}\left[\text { Xbest }_{i}+\alpha \times \operatorname{rand} \times \Delta X_{i}^{\text {new }}\right]
$$

Trong đó, round là hàm làm tròn đến số nguyên gần nhất, $\alpha>0$ là thông số bước di chuyển được chọn theo Yang [13], [20], rand là giá trị ngẫu nhiên trong khoảng [0,1] và gia số $\Delta X_{i}^{\text {new }}$ được xác định bởi biểu thức:

$$
\Delta X_{i}^{\text {new }}=\frac{\operatorname{rand}_{x}}{\left|\operatorname{rand}_{y}\right|^{1 / \beta}} \times \frac{\sigma_{x}(\beta)}{\sigma_{y}(\beta)} \times\left(\text { Xbest }_{i}-\text { Gbest }\right)
$$

Trong đó $\operatorname{rand}_{x}$ và $r a n d_{y}$ là hai biến phân phối ngẫu nhiên với độ lệch chuẩn $\sigma_{x}(\beta)$ và $\sigma_{y}(\beta)$. Hàm $\sigma_{x}(\beta)$ được tạo từ hàm phân phối gamma và $\sigma_{y}(\beta)=1$.

\section{walk \\ Buớc 3: Tạo ra giải pháp mới thông qua co chế phát hiện trứng la dụa trên phép di chuyển random}

Sau phép Lévy một quần thể mới được tạo ra và những cá thể tốt được cập nhật vào quần thể hiện tại. Tương tự như vậy, kết quả của phép random walk cũng là quần thể mới và các cá thể trong quần thể mới được tạo ra từ phép random walk sẽ được cập nhật vào quần thể hiện tại nếu hàm thích nghi của chúng tốt hơn hàm thích nghi của các cá thể trong quần thể hiện tại tương ứng. Quá trình tạo ra các giải pháp mới dựa trên phép random walk được thực hiện như sau.

$$
X_{i}^{\text {new }}=\operatorname{round}\left[\text { Xbest }_{i}+K(i,:) \otimes \Delta X_{i}^{\text {new }}\right]
$$

Trong đó $K$ là ma trận trong đó các phần tử bằng 0 hoặc 1 , được xác định bằng:

$$
K=\operatorname{rand}(N, D)>P_{a}
$$

Trong đó, $P a$ là xác suất phát hiện trứng lạ, được chọn bằng 0.2 .

Và gia số $\Delta X_{i}^{\text {new }}$ được xác định bởi: 


$$
\Delta X_{i}^{\text {new }}=\operatorname{rand} \times\left[\operatorname{randp}_{1}\left(\text { Xbest }_{i}\right)-\operatorname{randp}_{2}\left(\text { Xbest }_{i}\right)\right]
$$

Trong đó, rand là số ngẫu nhiên trong khoảng $[0,1]$, randp ${ }_{1}\left(\right.$ Xbest $\left._{i}\right)$ và $\operatorname{randp}_{2}\left(X_{\text {best }}\right)$ là nhiễu loạn ngẫu nhiên của vị trí các giải pháp trong quần thể.

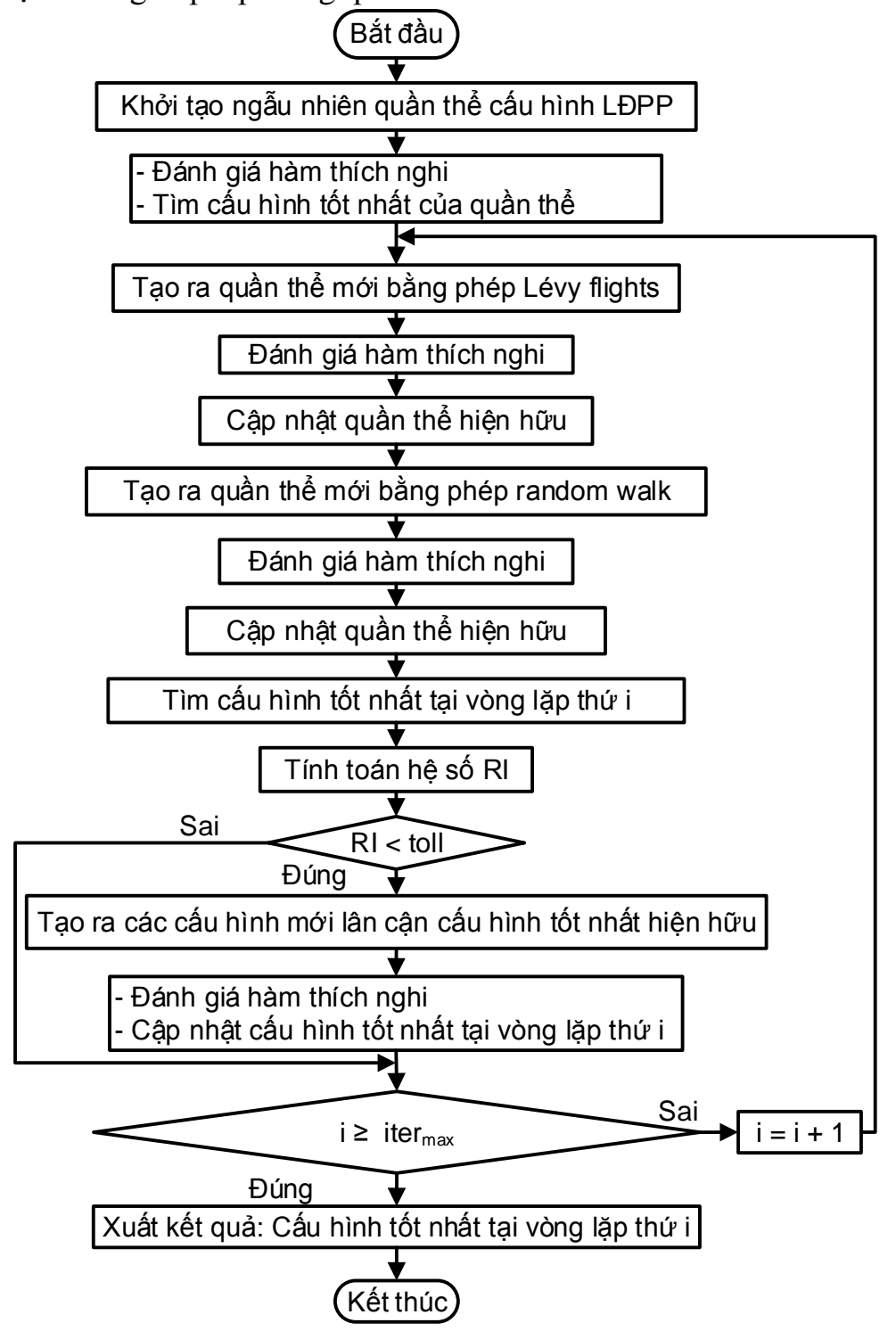

Hình 1: Thuật toán ECS cho bài toán tái cấu hình LĐPP.

Bước 4: Tìm kiếm cuc bộ bằng cơ chế cập nhật giải pháp tốt nhất hiện hưu

Do bởi bài toán tái cấu hình là bài toán rời rạc, việc thay đổi dù là một giá trị nhỏ các biến điều khiển (các khóa điện) trong một cấu hình có thể dẫn đến một cấu hình hoàn toàn mới. Điều này dẫn đến giá trị hàm mục tiêu sẽ rất khác biệt so với cấu hình hiện hữu. Như vậy, các thuật toán tối ưu nhất thiết phải được trang bị cơ chế tìm kiếm cục bộ sao cho có thể tìm kiếm được các cấu hình lân cận cấu hình tốt nhất hiện hữu để giúp thuật toán không bỏ sót các cấu hình lân cận cấu hình tốt nhất hiện hữu và nhanh chóng xác định được cấu hình tối ưu toàn cục.

Để thực hiện điều này, một cơ chế tìm kiếm cục bộ được sử dụng để trang bị bổ sung cho ECS để cập nhật lại cấu hình tốt nhất nếu phát hiện giải thuật đang rơi vào điểm tối ưu cục bộ. Nếu sau hai vòng lặp liên tiếp không có sự cải thiện trong giá trị hàm mục tiêu được xác định bằng biểu thức (9), cơ chế này được sử dụng để cập nhật lại cá thể tốt nhất hiện hữu.

$$
R I=f\left(X_{\text {gbest }}(i-1)\right)-f\left(X_{\text {gbest }}(i)\right)<\text { toll }
$$


Trong đó, toll là hằng số nhỏ để phân biệt được hai cấu hình so sánh là khác nhau và giá trị của toll được chọn bằng 0.01 .

Khi đó, từ cá thể tốt nhất, lần lượt có $D$ cá thể mới được tạo ra thông qua việc thay đổi lần lượt từng biến điều khiển của cá thể tốt nhất như biểu thức (10). Từ $D$ cá thể mới, giá trị hàm mục tiêu được tính toán và nếu có sự cải tiến trong giá trị hàm mục tiêu thì cá thể tốt nhất được cập nhật. Như vậy, cơ chế này không làm phát sinh thêm quần thể mới mà chỉ tạo ra một số cá thể xung quanh cá thể tốt nhất hiện hữu.

$$
X_{\text {new }, j}=\operatorname{round}\left[K I_{(j,:)} \times \mathrm{d} \times \operatorname{rand}+X_{\text {best }}(i)\right] \text { with } j=1,2, \ldots, D
$$

Trong đó, round là hàm làm tròn đến số nguyên gần nhất. KI là ma trận đơn vị. Để thay đổi giá trị một biến điều khiển trong $D$ biến của cá thể tốt nhất, giá trị $d$ được sử dụng để điều khiển khoảng cách từ biển hiện tại đến biến cập nhật. Giá trị $d$ này được lựa chọn dựa trên không gian của các biến điều khiển.

Bước 5: Điều kiện dùng giải thuật.

Điều kiện dừng của thuật toán là số vòng lặp đạt đến số vòng lặp thiết lập lớn nhất (iter ${ }_{\max }$ ). Lưu đồ thuật toán ECS được mô tả như Hình 1.

\section{KẾT QUẢ TÍNH TOÁN VÀ BÀN LUẬN}

\subsection{Kết quả tính toán}

Để đánh giá hiệu quả của phương pháp tái cấu hình LĐPP đề xuất, LĐPP 33 nút được sử dụng để tái cấu hình. LĐPP 33 nút có cấp điện áp $12.66 \mathrm{kV}$ bao gồm 37 nhánh, 32 khóa điện thường đóng và 5 khóa thường mở. Thông số nhánh và nút của lưới được tham khảo [21]. Tổng công suất của hệ thống là $3.72+$ j2.3 MVA. Sơ đồ đơn tuyến của hệ thống được cho ở Hình 2. Dòng điện định mức của các nhánh là 255 A [22].

Thuật toán ECS cho bài toán tái cấu hình LĐPP được xây dựng trong phần mềm Matlab phiên bản 2016a và chạy trên máy tính cá nhân có cấu hình Intel Core i5-2430M @2.4GHz, 4GB DDR3 RAM, Hard Drive 500GB, Windows 10 (64-bit). Thông số của ECS và CS được thiết lập dựa trên thực nghiệm như sau: Số lượng cá thể $N$ được đặt bằng 10 , số vòng lặp iter ${ }_{\max }$ lớn nhất 100 , khoảng cách từ giá trị của biến hiện tại đến giá trị của biến cập nhật $d$ được đặt bằng 4 .

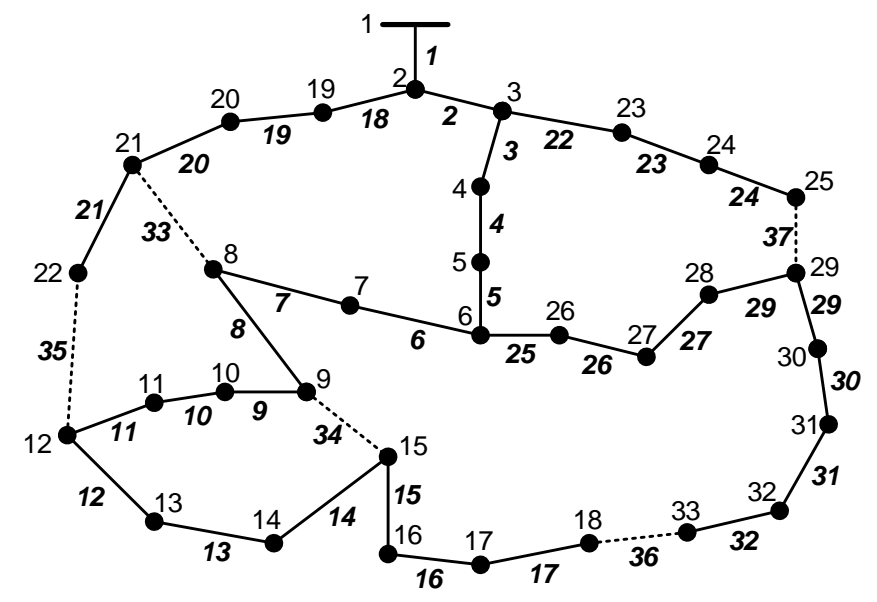

Hình 2: LĐPP 33 nút.

Kết quả tái cấu hình được trình trong Bảng 1 . Trong đó, kết quả tái cấu hình bằng giải thuật đề xuất ECS được so sánh với cấu trúc lưới ban đầu và kết quả tái cấu hình bằng giải thuật $\mathrm{CS}$. Ngoài ra, Bảng 1 cũng cũng trình bày kết quả quả so sánh giữa ECS với một số nghiên cứu đã thực hiện như FWA [23], GA [23], [24] và HSA [23], [24].

Các đặc tuyến hội tụ của ECS và CS trong 50 lần thực hiện độc lập được trình bày ở Hình 3. Các đường đặc tuyến hội tụ trung bình, lớn nhất và nhỏ nhất của $\mathrm{ECS}$ và $\mathrm{CS}$ trong các lần thực hiện đó được trình bày ở Hình 4 . Biên độ điện áp trước và sau khi tái cấu hình bằng ECS được trình bày ở Hình 5 . Hệ số mang tải trên các nhánh của lưới điện được trình bày ở Hình 6. 
Bảng 1: Kết quả so sánh phương pháp đề xuất $\mathrm{ECS}$ với $\mathrm{CS}$ và các phương pháp khác.

\begin{tabular}{|l|c|l|l|l|l|l|}
\hline Phương pháp & Ban đầu & ECS & CS & FWA [23] & $\begin{array}{l}\text { GA [23], } \\
{[24]}\end{array}$ & $\begin{array}{l}\text { HSA [23], } \\
{[24]}\end{array}$ \\
\hline Khóa mở & $\begin{array}{l}33,34, \\
35,36,37\end{array}$ & $\begin{array}{l}7,9,14, \\
32,37\end{array}$ & $\begin{array}{l}7,9,14, \\
32,37\end{array}$ & $\begin{array}{l}7,9,14, \\
28,32\end{array}$ & $\begin{array}{l}7,9,14, \\
32,37\end{array}$ & $\begin{array}{l}7,10,14, \\
36,37\end{array}$ \\
\hline Tổn thất (kW) & 202.69 & 139.5543 & 139.5543 & 139.98 & 139.55 & 142.6816 \\
\hline $\begin{array}{l}\text { Giá trị lớn nhất hàm } \\
\text { thích nghi }\end{array}$ & - & 140.7726 & 147.2806 & 155.75 & 202.67 & 195.10 \\
\hline $\begin{array}{l}\text { Giá trị nhỏ nhất hàm } \\
\text { thích nghi }\end{array}$ & - & 140.7726 & 140.7726 & 140.8580 & 140.7726 & 144.3231 \\
\hline $\begin{array}{l}\text { Giá trị trung bình hàm } \\
\text { thích nghi }\end{array}$ & - & 140.7726 & 141.3288 & 145.63 & 166.2 & 152.33 \\
\hline $\begin{array}{l}\text { Độ lệch chuấn hàm thích } \\
\text { nghi }\end{array}$ & - & 0 & 1.3026 & 5.49 & 14.53 & 11.28 \\
\hline $\begin{array}{l}\text { Số lần thành công/số lần } \\
\text { chạy }\end{array}$ & - & $50 / 50$ & $33 / 50$ & - & - & - \\
\hline $\begin{array}{l}\text { Vòng lặp hội tụ trung } \\
\text { bình }\end{array}$ & - & 25 & 40 & - & - & - \\
\hline Thời gian thực hiện (s) & - & 9.5984 & 7.9653 & - & - & - \\
\hline
\end{tabular}

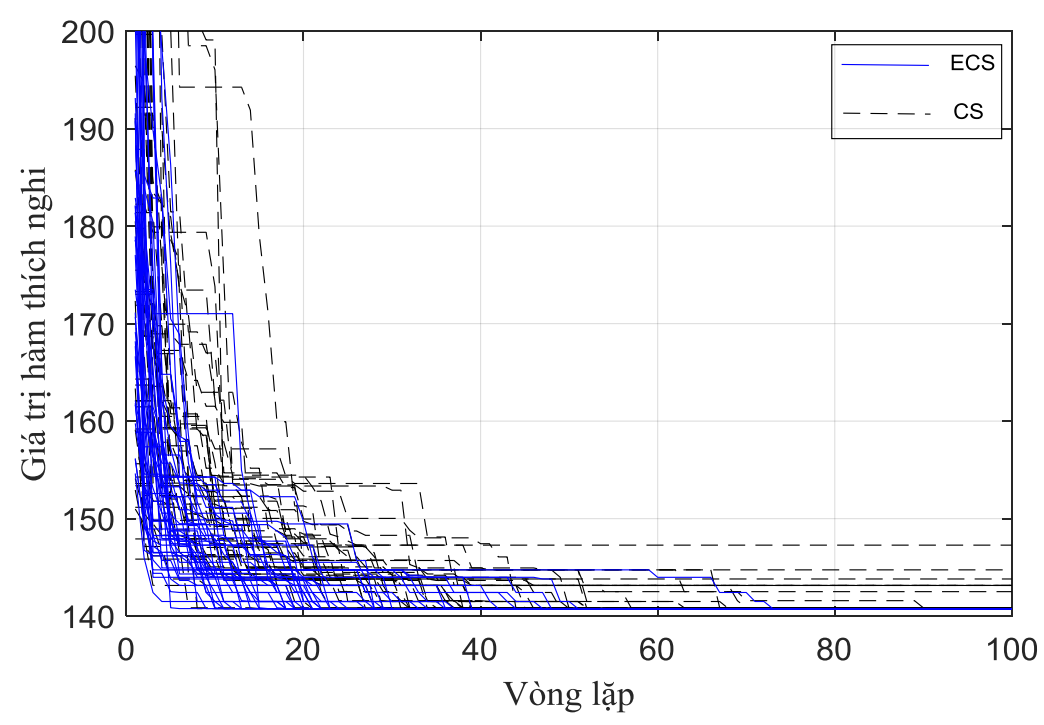

Hình 3: Đặc tuyến hội tụ của ECS và CS trong 50 lần chạy độc lập. 
TỐI UUU CẤU HÌNH LUOÓI ĐIỆN PHÂN PHỐI GIẢM TỔN THÂT CÔNG SUÂT SỬ DỤNG THUẠT TOÁN CUCKOO SEARCH CẢI TIẾN

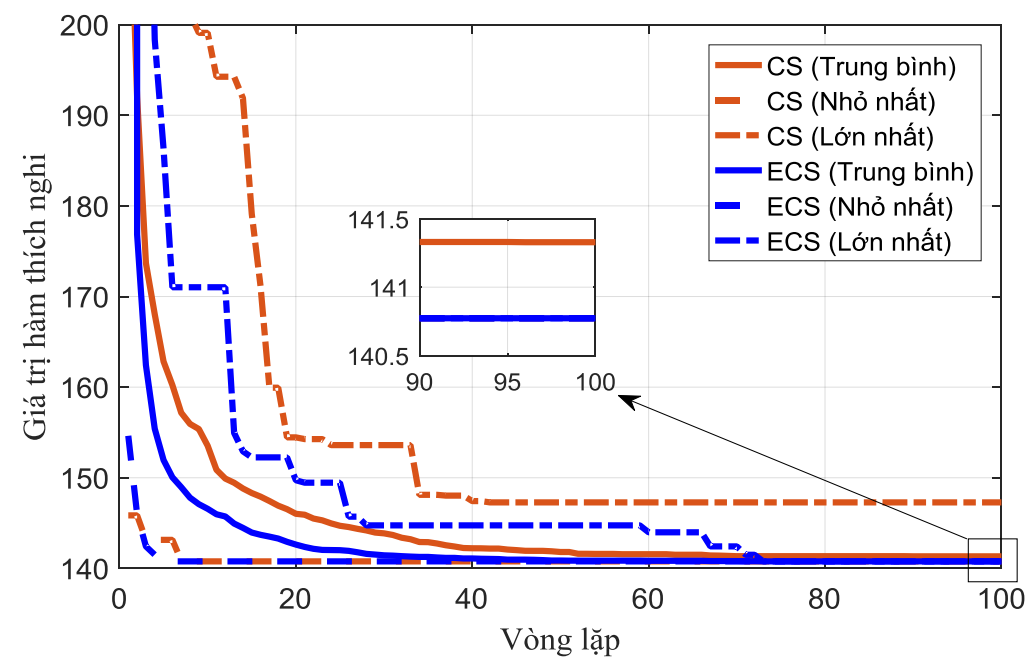

Hình 4: Đặc tuyến hội tụ lớn nhất, nhỏ nhất và trung bình của ECS và CS trong 50 lần chạy độc lập.

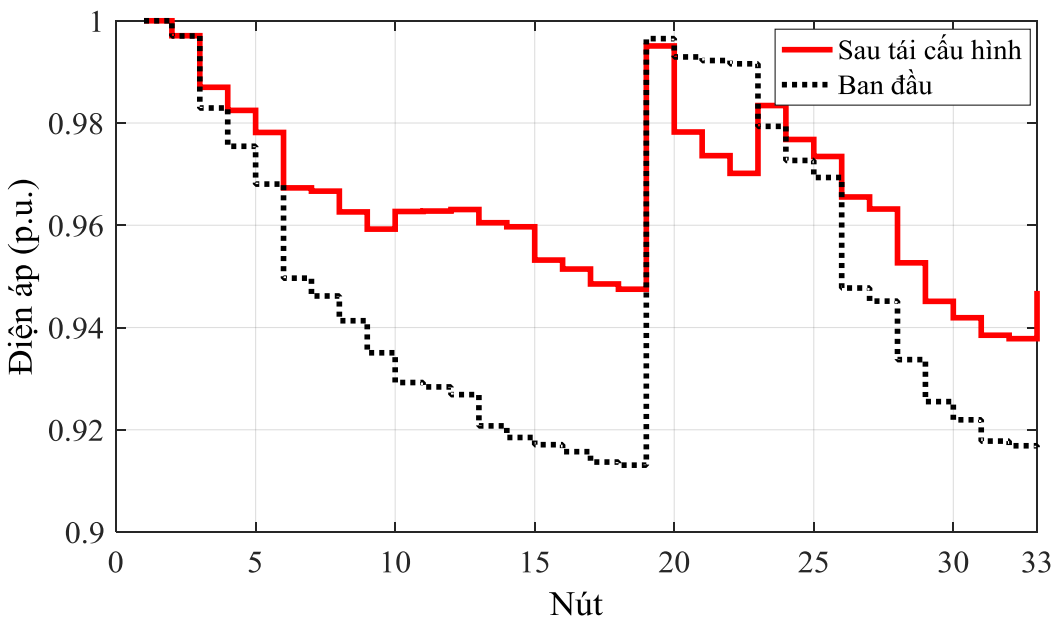

Hình 5: Biên độ điện áp các nút trước và sau khi tái cấu hình.

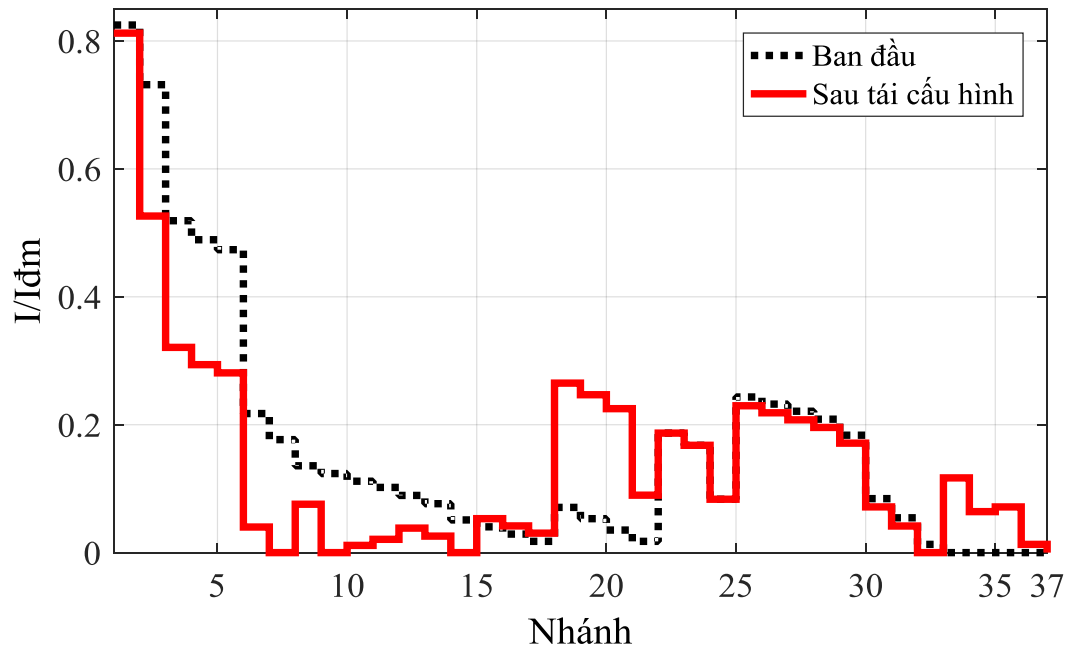

Hình 6: Hệ số mang tải của các nhánh truớc và sau khi tái cấu hình. 


\subsection{Bàn luận}

Từ kết quả được tái cấu hình được trình bày ở mục 4.1 có thể thấy được ưu điểm vượt trội của ECS so với $\mathrm{CS}$ cũng như một số phương pháp khác. Cụ thể như sau:

Bảng 1 cho thấy mặc dù cả ECS và $C S$ đều tìm được cấu hình LĐPP tối ưu với các khóa điện mở $\{7$, $9,14,32,37$ và tồn thất công suất tương ứng $139.5543 \mathrm{~kW}$ nhưng giá trị trung bình sau 50 lần chạy độc lập cho thấy ECS có nhiều ưu điểm hơn so với CS. Cụ thể, trong 50 lần thực hiện ECS tìm được cấu hình tối ưu trong tất cả các lần chạy, trong khi CS chỉ tìm được cấu trúc tối ưu trong 33 lần chạy độc lập thấp hơn $34 \%$ so với ECS. Giá trị thích nghi trung bình của ECS là 140.7726 so với 141.3288 thu được từ $\mathrm{CS}$. Độ lệch chuẩn của hàm thích nghi của ECS là 0 nhỏ hơn 1.3026 so với CS. Đặc biệt số vòng lặp hội tụ trung bình của ECS nhỏ hơn nhiều so với CS. Trong các lần thực hiện, ECS hội tụ trong khoảng 25 vòng lặp, trong khi CS thường hội tụ sau 40 vòng lặp. Điểu này cho thấy, nhờ có có cơ chế tìm kiếm cục bộ mà ECS có khả năng tìm được cấu trúc tôi ưu với tỉ lệ thành công lớn hơn và số vòng lặp nhỏ hơn so với CS. Thời gian tính toán trung bình của ECS cao hơn $1.6331 \mathrm{~s}$. Tuy nhiên, do ECS có khả năng tìm được cấu trúc tối ưu với số vòng lặp nhỏ nên để rút ngắn thời gian tính toán của ECS bằng cách thiết lập số vòng lặp nhỏ hơn so với CS. Hình 3 cho thấy trong tất cả các lần thực hiện ECS đều hội tụ đến cùng một giá trị tối ưu với số vòng lặp nhỏ hơn nhiều so với CS. Các đường đặc tuyến hội tụ trung bình, lớn nhất và nhỏ nhất ở Hình 4 chứng tỏ rằng ECS vượt trội về khả năng hội tụ đến giá trị tối ưu toàn cục so với $\mathrm{CS}$. Đường đặc tuyến trung bình của ECS gần với đường đặc tuyến nhỏ nhất hơn so với CS. Điều này chứng minh sự ổn định và độ tin cậy của kết quả thực hiện trong các lần thực hiện của ECS so với CS. Ngoài ra, sau khi thực hiện tái cấu hình bằng ECS, biên độ điện áp các nút (được trình bày ở Hình 5 ) được cải thiện đáng kể so với trước khi thực hiện tái cấu hình. Hệ số mang tải trên các nhánh (được trình bày ở Hình 6 ) cho thấy không có nhánh nào bị quá tải.

Bảng 1 cũng cho thấy, sau khi thực hiện tái cấu hình, tổn thất công suất giảm từ $202.69 \mathrm{~kW}$ xuống $139.55 \mathrm{~kW}$. Kết quả này hoàn toàn tương tự với kết quả thực hiện bằng các phương pháp GA và tốt hơn kết quả thực hiện bằng các phương pháp FWA và HSA. Có thể thấy rằng, FWA thu được cấu trúc lưới $\{7,9,14,28,32\}$ với tổn thất $139.98 \mathrm{~kW}$, Cấu hình này chỉ khác một khóa điện so với cấu hình tối ưu toàn cục. Điều này cho thấy cơ chế khai thác đề xuất có ý nghĩa khi giải bài toán tái cấu hình LĐPP.

\section{KẾT LUẬn}

Bài báo trình bày phương pháp tái cấu hình LĐPP giảm tổn thất công suất dựa trên giải thuật $\mathrm{CS}$ cải tiến. Để áp nâng cao hiệu quả của thuật toán CS khi giải bài toán tái cấu hình, cơ chế tìm kiếm cục bộ được trang bị để khải thác không gian tìm kiếm lân cận các giải pháp tối ưu hiện hữu. Để chứng minh hiệu quả của thuật toán, lưới điện 33 nút được sử dụng để tái cấu hình. Kết quả so sánh giữa ECS và CS cho thấy ECS có nhiều điểm vượt trội so với CS ở chất lượng giải pháp thu được với tỉ lệ thành công trong 50 lần thực hiện cao hơn 34\%, các giá trị lớn nhất và trung bình của hàm thích nghi thu được bé hơn so với CS, số vòng lặp hội tụ trung bình đến giải pháp tối ưu của ECS thấp hơn $38 \%$ so với CS. Vì vậy, ECS là thuật toán tin cậy và hứa hẹn để áp dụng cho bài toán tái cấu hình giảm tổn thất công suất nói riêng và các bài toán tái cấu hình thỏa mãn các mục tiêu khác nói chung.

\section{TÀI LIỆU THAM KHẢO}

[1] S. Gopiya Naik, D. K. Khatod, and M. P. Sharma, "Optimal allocation of combined DG and capacitor for real power loss minimization in distribution networks,” Int. J. Electr. Power Energy Syst., vol. 53, pp. 967-973, 2013.

[2] A. Merlin and H. Back, "Search for a minimal loss operating spanning tree configuration in an urban power distribution system," Proceeding 5th power Syst. Comput. conf (PSCC), Cambridge, UK, vol. 1-18, 1975.

[3] S. Civanlar, J. J. Grainger, H. Yin, and S. S. H. Lee, "Distribution feeder reconfiguration for loss reduction," IEEE Trans. Power Deliv., vol. 3, no. 3, pp. 1217-1223, 1988. 
[4] J. Z. Zhu, "Optimal reconfiguration of electrical distribution network using the refined genetic algorithm," Electr. Power Syst. Res., vol. 62, no. 1, pp. 37-42, 2002.

[5] R. T. Ganesh Vulasala, Sivanagaraju Sirigiri, "Feeder Reconfiguration for Loss Reduction in Unbalanced Distribution System Using Genetic Algorithm,” Int. J. Electr. Electron. Eng., vol. 3, no. 12, pp. 754-762, 2009.

[6] P. Subburaj, K. Ramar, L. Ganesan, and P. Venkatesh, "Distribution System Reconfiguration for Loss Reduction using Genetic Algorithm,” J. Electr. Syst., vol. 2, no. 4, pp. 198-207, 2006.

[7] K. K. Kumar, N. Venkata, and S. Kamakshaiah, "FDR particle swarm algorithm for network reconfiguration of distribution systems," J. Theor. Appl. Inf. Technol., vol. 36, no. 2, pp. 174-181, 2012.

[8] T. M. Khalil and A. V Gorpinich, "Reconfiguration for Loss Reduction of Distribution Systems Using Selective Particle Swarm Optimization,” Int. J. Multidiscip. Sci. Eng., vol. 3, no. 6, pp. 16-21, 2012.

[9] S. F. Mekhamer, F. M. Mohammed, and M. a L. Badr, "A Modified Particle Swarm Technique for Distribution Systems Reconfiguration," online J. Electron. Electr. Eng., vol. 1, no. 1, pp. 121-129.

[10] R. Pegado, Z. Ñaupari, Y. Molina, and C. Castillo, "Radial distribution network reconfiguration for power losses reduction based on improved selective BPSO," Electr. Power Syst. Res., vol. 169, no. December 2018, pp. 206-213, 2019.

[11] A. Onlam, D. Yodphet, R. Chatthaworn, C. Surawanitkun, A. Siritaratiwat, and P. Khunkitti, "Power Loss Minimization and Voltage Stability Improvement in Electrical Distribution System via Network Reconfiguration and Distributed Generation Placement Using Novel Adaptive Shuffled Frogs Leaping Algorithm," Energies, vol. 12, no. 3, p. 553, 2019.

[12] A. Roosta, H.-R. Eskandari, and M.-H. Khooban, "Optimization of radial unbalanced distribution networks in the presence of distribution generation units by network reconfiguration using harmony search algorithm," Neural Comput. Appl., vol. 0, 2018.

[13] X. S. Yang and S. Deb, “Cuckoo search via Lévy flights,” in 2009 World Congress on Nature and Biologically Inspired Computing, NABIC 2009 - Proceedings, 2009, pp. 210-214.

[14] T. T. Nguyen, D. N. Vo, and A. V. Truong, "Cuckoo search algorithm for short-term hydrothermal scheduling," Appl. Energy, vol. 132, pp. 276-287, 2014.

[15] T. T. Nguyen, D. N. Vo, and B. H. Dinh, "Cuckoo search algorithm for combined heat and power economic dispatch,” Int. J. Electr. Power Energy Syst., vol. 81, pp. 204-214, 2016.

[16] K. Chandrasekaran and S. P. Simon, "Multi-objective scheduling problem: Hybrid approach using fuzzy assisted cuckoo search algorithm," Swarm Evol. Comput., vol. 5, pp. 1-16, 2012.

[17] T. T. Nguyen and A. V. Truong, "Distribution network reconfiguration for power loss minimization and voltage profile improvement using cuckoo search algorithm,” Int. J. Electr. Power Energy Syst., vol. 68, pp. 233-242, 2015.

[18] Z. Moravej and A. Akhlaghi, "A novel approach based on cuckoo search for DG allocation in distribution network,” Int. J. Electr. Power Energy Syst., vol. 44, no. 1, pp. 672-679, 2013.

[19] O. P. Mahela, S. Ram, and O. Lalit, "Optimal Capacitor Placement for Loss Reduction in Radial Distribution Feeder," Mod. Electr. Power Syst., vol. 4, no. 6, pp. 43-48, 2013. 
[20] X. S. Yang and S. Deb, "Cuckoo search: Recent advances and applications," Neural Comput. Appl., vol. 24, no. 1, pp. 169-174, 2014.

[21] M. E. Baran and F. F. Wu, "Network reconfiguration in distribution systems for loss reduction and load balancing," IEEE Transactions on Power Delivery, vol. 4, no. 2. pp. 1401-1407, 1989.

[22] S. Ghasemi and J. Moshtagh, "Radial distribution systems reconfiguration considering power losses cost and damage cost due to power supply interruption of consumers," Int. J. Electr. Eng. Informatics, vol. 5, no. 3, pp. 297-315, 2013.

[23] A. Mohamed Imran and M. Kowsalya, "A new power system reconfiguration scheme for power loss minimization and voltage profile enhancement using Fireworks Algorithm," Int. J. Electr. Power Energy Syst., vol. 62, pp. 312-322, 2014.

[24] R. S. Rao, S. Venkata, L. Narasimham, M. R. Raju, and a S. Rao, "Optimal Network Reconfiguration of Large-Scale Distribution System Using Harmony Search Algorithm,” IEEE Trans. Power Syst., vol. 26, no. 3, pp. 1080-1088, 2011.

Ngày nhận bài:28/05/2019

Ngày chấp nhận đăng:06/08/2019 